\title{
THE CRITICAL VALUES OF $L$-FUNCTIONS OF CM-BASE CHANGE FOR HILBERT MODULAR FORMS
}

\author{
CRISTIAN VIRDOL
}

\begin{abstract}
In this paper we generalize some results, obtained by Shimura, on the critical values of $L$-functions of $l$-adic representations attached to quadratic CM-base change of Hilbert modular forms twisted by finite order characters, to the case of the critical values of $L$-functions of arbitrary base change to CM-number fields of $l$-adic representations attached to Hilbert modular forms twisted by some finite-dimensional representations.
\end{abstract}

Keywords: critical values, CM-fields, Hilbert modular forms.

\section{Introduction}

For $F$ a totally real number field, let $J_{F}$ be the set of infinite places of $F$, and let $\Gamma_{F}:=\operatorname{Gal}(\overline{\mathbb{Q}} / F)$. Let $f$ be a normalized Hecke eigenform of $\operatorname{GL}(2) / F$ of weight $k=(k(\tau))_{\tau \in J_{F}}$, where all $k(\tau)$ have the same parity and $k(\tau) \geqslant 2$. We denote by $\Pi$ the cuspidal automorphic representation of GL(2)/ $F$ generated by $f$. In this paper we assume that $\Pi$ is non-CM. We denote by $\rho_{\Pi}$ the $l$-adic representation attached to $\Pi$, for some prime number $l$ (by fixing an isomorphism $\iota: \overline{\mathbb{Q}}_{l} \stackrel{\sim}{\longrightarrow} \mathbb{C}$ one can regard $\rho_{\Pi}$ as a complex-valued representation). Define $k_{0}=\max \left\{k(\tau) \mid \tau \in J_{F}\right\}$ and $k^{0}=\min \left\{k(\tau) \mid \tau \in J_{F}\right\}$. Any integer $m \in \mathbb{Z}$ such that $\left(k_{0}-k^{0}\right) / 2<m<$ $\left(k_{0}+k^{0}\right) / 2$ is called a critical point for $f$ or $\Pi$. Throughout this paper we write $a \sim b$ for $a, b \in \mathbb{C}$ if $b \neq 0$ and $a / b \in \overline{\mathbb{Q}}$.

In this article we prove the following result.

Theorem 1.1. Assume $k(\tau) \geqslant 3$ for all $\tau \in J_{F}$, and $k(\tau)$ mod 2 is independent of $\tau$. Let $M$ be a finite $C M$-extension of $F$, and let $\Gamma_{M}:=\operatorname{Gal}(\overline{\mathbb{Q}} / M)$. Assume that $\chi$ is a continuous complex-valued abelian representation of $\Gamma_{M}$, and that $\phi$ is a continuous complex-valued representation of $\Gamma_{M}$ satisfyng the following property: $K:=\overline{\mathbb{Q}}^{\text {ker } \phi}$ is a $(\mathbb{Z} / 2 \mathbb{Z})^{r}$-extension of a CM-number field for some non-negative $11 \mathrm{R} 80$

2010 Mathematics Subject Classification: primary: 11F41; secondary: 11F80, 11R42, 
integer $r$. Let $\psi=\phi \otimes \chi$. Then

$$
L\left(m,\left.\iota \rho_{\Pi}\right|_{\Gamma_{M}} \otimes \psi\right) \sim \pi^{\left(m+1-k_{0}\right)[M: \mathbb{Q}] \operatorname{dim} \psi}\langle f, f\rangle^{\frac{[M: F]}{2} \operatorname{dim} \psi},
$$

for any integer $m$ satisfying

$$
\left(k_{0}+1\right) / 2 \leqslant m<\left(k_{0}+k^{0}\right) / 2 .
$$

Theorem 1.1 is a generalization of Theorem 5.7 of [S1] (i.e. Proposition 2.1 below; the inner product $\langle f, f\rangle$ is normalized as in $\S 2$ below).

\section{Known results}

Consider $F$ a totally real number field and let $J_{F}$ be the set of infinite places of $F$. If $\Pi$ is a cuspidal automorphic representation (discrete series at infinity) of weight $k=(k(\tau))_{\tau \in J_{F}}$ of $\mathrm{GL}(2) / F$, where all $k(\tau)$ have the same parity and all $k(\tau) \geqslant 2$, let $k_{0}=\max \left\{k(\tau) \mid \tau \in J_{F}\right\}$ and $k^{0}=\min \left\{k(\tau) \mid \tau \in J_{F}\right\}$. Let $O$ be the coefficient ring of $\Pi$ (i.e. $O$ is the ring of integers of the field generated over $\mathbb{Q}$ by the eigenvalues $a_{\wp}$ defined by $T_{\wp} f=a_{\wp} f$, where $T_{\wp}$ is the Hecke operator at $\wp$, and $\wp$ runs over the prime ideals of $F$ ), and let $\lambda$ be a prime ideal of $O$ above some rational prime $l$. Then there exists $([\mathrm{T}])$ a $\lambda$-adic representation

$$
\rho_{\Pi}:=\rho_{\Pi, \lambda}: \Gamma_{F} \rightarrow \mathrm{GL}_{2}\left(O_{\lambda}\right) \hookrightarrow \mathrm{GL}_{2}\left(\overline{\mathbb{Q}}_{l}\right),
$$

which satisfies $L\left(s, \iota \rho_{\Pi, \lambda}\right)=L\left(s-\frac{\left(k_{0}-1\right)}{2}, \Pi\right)=L\left(s-\frac{\left(k_{0}-1\right)}{2}, f\right)$, where $\iota: \overline{\mathbb{Q}}_{l} \stackrel{\sim}{\longrightarrow}$ $\mathbb{C}$ is a specific isomorphism (the above equality of $L$-functions is up to finitely many Euler factors), and the representation $\rho_{\Pi}$ is unramified outside the primes dividing $\mathbf{n} l$. Because the line of convergence of $L(s, \Pi)$ is $\operatorname{Re}(s)=1$, we get that the line of convergence of $L\left(s, \rho_{\Pi, \lambda}\right)$ is $\operatorname{Re}(s)=\left(k_{0}+1\right) / 2$. Here $f$ is the normalized Hecke eigenform of $\mathrm{GL}(2) / F$ of weight $k$ corresponding to $\Pi, \mathbf{n}$ is the level of $\Pi$. We define

$$
\langle f, f\rangle=\pi^{\sum_{\tau \in J_{F}} k(\tau)} \int_{Z_{\infty+} \mathrm{GL}_{2}(F) \backslash \mathrm{GL}_{2}\left(\mathbb{A}_{F}\right)} f(x) \overline{f(x)} d x
$$

where $Z_{\infty+} \simeq \mathbb{R}_{+}^{\times}$is the connected component of the center of $\mathrm{GL}_{2}(\mathbb{R})$, and the measure is normalized such that $\operatorname{vol}\left(Z_{\infty+} \mathrm{GL}_{2}(F) \backslash \mathrm{GL}_{2}\left(\mathbb{A}_{F}\right)\right)=1$.

We know (by Proposition 5.2 and Theorem 5.7 of [S1]; we actually use the fact that $\left.L\left(s,\left.\iota \rho_{\Pi}\right|_{\Gamma_{M}} \otimes \psi\right)=L\left(s, \iota \rho_{\Pi} \otimes \operatorname{Ind}_{\Gamma_{M}}^{\Gamma_{F}} \psi\right)\right)$ in order to reduce Proposition 2.1 below to a particular case of Theorem 5.7 of [S1] where a convolution of two cuspidal automorphic representations (one non-CM, and the other $\mathrm{CM}$ ) of $\mathrm{GL}(2) / F$ was considered; we remark that $\operatorname{Ind}_{\Gamma_{M}}^{\Gamma_{F}} \psi$ corresponds to a $\mathrm{CM}$ cuspidal automorphic representation of $\mathrm{GL}(2) / F$ of weight 1$)$.

Proposition 2.1. Assume $k(\tau) \geqslant 2$ for all $\tau \in J_{F}$ and $k(\tau) \bmod 2$ is independent of $\tau$. Let $M$ be a quadratic CM-extension of $F$, and let $\psi$ be a continuous onedimensional representation of $\Gamma_{M}$. Then

$$
L\left(m,\left.\iota \rho_{\Pi}\right|_{\Gamma_{M}} \otimes \psi\right) \sim \pi^{\left(m+1-k_{0}\right)[M: \mathbb{Q}]}\langle f, f\rangle
$$


for any integer $m$ satisfying

$$
\left(k_{0}+1\right) / 2 \leqslant m<\left(k_{0}+k^{0}\right) / 2 .
$$

\section{The proof of Theorem 1.1 for $\psi$ a character}

We fix a non-CM cuspidal automorphic representation $\Pi$ of GL(2)/F as in Theorem 1.1, and let $M / F$ be a finite CM-extension. In this section we assume that $\psi$ is an arbitrary one-dimensional continuous representation of $\Gamma_{M}$ and prove Theorem 1.1 in this case.

We know the following result (Theorem 1.1 of [V1], or Theorem 2.1 of [V2], or Theorem A of [BGGT]):

Theorem 3.1. Let $\Pi$ be a cuspidal automorphic representation of weight $k=$ $(k(\tau))_{\tau \in J_{F}}$ of $G L(2) / F$, where all $k(\tau)$ have the same parity and all $k(\tau) \geqslant 2$. Let $F^{\prime}$ be a totally real extension of $F$. Then there exists a totally real Galois extension $F^{\prime \prime}$ of $F^{\prime}$, such that $\left.\rho_{\Pi}\right|_{\Gamma_{F^{\prime \prime}}}$ is cuspidal automorphic i.e. there exists a cuspidal automorphic representation $\Pi^{\prime \prime}$ of weight $k^{\prime \prime}$ of $G L(2) / F^{\prime \prime}$ such that $\left.\rho_{\Pi}\right|_{\Gamma_{F^{\prime \prime}}} \cong \rho_{\Pi^{\prime \prime}}$.

We denote by $F^{\prime}$ the maximal totally real subfield of $M$; hence $M$ is a quadratic CM-extension of $F^{\prime}$. Then from Theorem 3.1 we know that we can find a totally real Galois extension $F^{\prime \prime}$ of $F^{\prime}$, and a cuspidal automorphic representation $\Pi^{\prime \prime}$ of $\mathrm{GL}(2) / F^{\prime \prime}$ such that $\left.\rho_{\Pi}\right|_{\Gamma_{F^{\prime \prime}}} \cong \rho_{\Pi^{\prime \prime}}$. Because $\Pi$ is non-CM, we get that $\Pi^{\prime \prime}$ is non-CM.

From Theorem 15.10 of $[\mathrm{CR}]$ we know that there exist some subfields $M_{i} \subseteq$ $M F^{\prime \prime}$ such that $M \subseteq M_{i}$ and $\operatorname{Gal}\left(M F^{\prime \prime} / M_{i}\right)$ are solvable, and some integers $n_{i}$, such that the trivial representation

$$
1_{M}: \operatorname{Gal}\left(M F^{\prime \prime} / M\right) \rightarrow \mathbb{C}^{\times}
$$

can be written as

$$
1_{M}=\sum_{i=1}^{u} n_{i} \operatorname{Ind}_{\operatorname{Gal}\left(M F^{\prime \prime} / M_{i}\right)}^{\operatorname{Gal}\left(M F^{\prime \prime} / M\right)} 1_{M_{i}},
$$

(an equality in the character ring of $\operatorname{Gal}\left(M F^{\prime \prime} / M\right)$ ), where $1_{M_{i}}: \operatorname{Gal}\left(M F^{\prime \prime} / M_{i}\right) \rightarrow$ $\mathbb{C}^{\times}$is the trivial representation. In particular we have $1=\sum_{i=1}^{u} n_{i}\left[M_{i}: M\right]$. Then

$$
\begin{aligned}
L\left(s,\left.\iota \rho_{\Pi}\right|_{\Gamma_{M}} \otimes \psi\right) & =\prod_{i=1}^{u} L\left(s,\left.\iota \rho_{\Pi}\right|_{\Gamma_{M}} \otimes \operatorname{Ind}_{\Gamma_{M_{i}}}^{\Gamma_{M}} 1_{M_{i}} \otimes \psi\right)^{n_{i}} \\
& =\prod_{i=1}^{u} L\left(s, \operatorname{Ind}_{\Gamma_{M_{i}}}^{\Gamma_{M}}\left(\left.\iota \rho_{\Pi}\right|_{\Gamma_{M_{i}}}\right) \otimes \psi\right)^{n_{i}} \\
& =\prod_{i=1}^{u} L\left(s,\left.\left.\iota \rho_{\Pi}\right|_{\Gamma_{M_{i}}} \otimes \psi\right|_{\Gamma_{M_{i}}}\right)^{n_{i}} .
\end{aligned}
$$


Since $\left.\rho_{\Pi}\right|_{\Gamma_{F^{\prime \prime}}}$ is cuspidal automorphic and $M F^{\prime \prime}$ is a quadratic extension of $F^{\prime \prime}$ we get that $\left.\rho_{\Pi}\right|_{\Gamma_{M F^{\prime \prime}}}$ is cuspidal automorphic, and because $\operatorname{Gal}\left(M F^{\prime \prime} / M_{i}\right)$ is solvable, one gets easily (see $\S 4$ of [V4]) that $\left.\rho_{\Pi}\right|_{\Gamma_{M_{i}}}$ is cuspidal automorphic.

Hence the function $L\left(s,\left.\iota \rho_{\Pi}\right|_{\Gamma_{M}} \otimes \psi\right)$ has a meromorphic continuation to the entire complex plane and satisfies a functional equation because each function $L\left(s,\left.\left.\iota \rho_{\Pi}\right|_{\Gamma_{M_{i}}} \otimes \psi\right|_{\Gamma_{M_{i}}}\right)$ has a meromorphic continuation to the entire complex plane and satisfies a functional equation. Moreover, since each function $L\left(s, \iota \rho_{\Pi} \mid \Gamma_{M_{i}} \otimes\right.$ $\left.\psi\right|_{\Gamma_{M_{i}}}$ ) has no poles or zeros for $\operatorname{Re}(s) \geqslant\left(k_{0}+1\right) / 2$ (see Proposition 5.2 of [S1] and Proposition 4.16 of [S2]), we get that the function $L\left(s, \iota \rho_{\Pi} \mid \Gamma_{M} \otimes \psi\right)$ has no poles or zeros for $\operatorname{Re}(s) \geqslant\left(k_{0}+1\right) / 2$. Thus for any integer $m$ satisfying

$$
k_{0}+1 \leqslant m
$$

we get the identity

$$
L\left(m,\left.\iota \rho_{\Pi}\right|_{\Gamma_{M}} \otimes \psi\right)=\prod_{i=1}^{u} L\left(m,\left.\left.\iota \rho_{\Pi}\right|_{\Gamma_{M_{i}}} \otimes \psi\right|_{\Gamma_{M_{i}}}\right)^{n_{i}} .
$$

Let $F_{i}$ be the maximal totally real subfield of $M_{i}$. Since $\left.\rho_{\Pi}\right|_{\Gamma_{M_{i}}}$ is cuspidal automorphic and $M_{i} / F_{i}$ is quadratic, one can prove easily that $\left.\rho_{\Pi}\right|_{\Gamma_{F_{i}}}$ is cuspidal automorphic (see Lemma 1.3 of $[\mathrm{BGHT}]$ ), so $\left.\rho_{\Pi}\right|_{\Gamma_{F_{i}}} \cong \rho_{\Pi_{i}}$ for some cuspidal automorphic representation $\Pi_{i}$ of $\mathrm{GL}(2) / F_{i}$. We denote by $f_{i}$ the normalized Hecke eigenform of $\mathrm{GL}(2) / F_{i}$ associated to $\Pi_{i}$. Then $f_{i}$ has weight $k_{i}=\left(k_{i}(\tau)\right)_{\tau \in J_{F_{i}}}$, where $J_{F_{i}}$ is the set of infinite places of $F_{i}$, and $k_{i}(\tau)=k(\tau \mid F)$ for any $\tau \in J_{F_{i}}$.

Now from Proposition 2.1 we get that

$$
L\left(m,\left.\left.\iota \rho_{\Pi}\right|_{\Gamma_{M_{i}}} \otimes \psi\right|_{\Gamma_{M_{i}}}\right) \sim \pi^{\left(m+1-k_{0}\right)\left[M_{i}: \mathbb{Q}\right]}\left\langle f_{i}, f_{i}\right\rangle,
$$

for any integer $m$ satisfying

$$
\left(k_{0}+1\right) / 2 \leqslant m<\left(k_{0}+k^{0}\right) / 2 .
$$

But we know that (see the paragraph just before Remark 5.1 of [V3])

$$
\left\langle f_{i}, f_{i}\right\rangle \sim\langle f, f\rangle^{\left[F_{i}: F\right]},
$$

and using the fact that $1=\sum_{i=1}^{u} n_{i}\left[M_{i}: M\right]$, we obtain

$$
\begin{aligned}
L\left(m,\left.\iota \rho_{\Pi}\right|_{\Gamma_{M}} \otimes \psi\right) & \sim \pi^{\sum_{i=1}^{u}\left(m+1-k_{0}\right)\left[M_{i}: \mathbb{Q}\right] n_{i}} \prod_{i=1}^{u}\left\langle f_{i}, f_{i}\right\rangle^{n_{i}} \\
& \sim \pi^{\sum_{i=1}^{u}\left(m+1-k_{0}\right)\left[M_{i}: \mathbb{Q}\right] n_{i}}\langle f, f\rangle^{\sum_{i=1}^{u}\left[F_{i}: F\right] n_{i}} \\
& \sim \pi^{\left(m+1-k_{0}\right)[M: \mathbb{Q}]}\langle f, f\rangle^{\frac{[M: F]}{2}},
\end{aligned}
$$

for any integer $m$ satisfying

$$
\left(k_{0}+1\right) / 2 \leqslant m<\left(k_{0}+k^{0}\right) / 2,
$$

which proves Theorem 1.1 for $\psi$ a one-dimensional representation. 


\section{The proof of Theorem 1.1 for general $\psi$}

Let $\psi=\phi \otimes \chi$ be a finite-dimensional representation of $\Gamma_{M}$ as in Theorem 1.1. We denote by $M^{\prime}$ the maximal CM-subfield of $K:=\overline{\mathbb{Q}}^{\text {ker } \phi}$ which contains $M$. Applying the conditions in Theorem 1.1 , we see that $K$ is a $(\mathbb{Z} / 2 \mathbb{Z})^{r}$-extension of $M^{\prime}$ for some $r$, and $\chi$ is a direct sum of one-dimensional representations.

From the beginning of $\S 15$ of $[\mathrm{CR}]$ we know that there exist some subfields $E_{j} \subseteq K$ such that $M \subseteq E_{j}$ and $\operatorname{Gal}\left(K / E_{j}\right)$ are solvable (actually we don't use this solvability), and some integers $m_{j}$, such that the representation

$$
\phi: \operatorname{Gal}(K / M) \rightarrow \mathrm{GL}_{N}(\mathbb{C})
$$

can be written as

$$
[K: M] \phi=\sum_{j=1}^{v} m_{j} \operatorname{Ind}_{\operatorname{Gal}\left(K / E_{j}\right)}^{\operatorname{Gal}(K / M)} 1_{E_{j}},
$$

where $1_{E_{j}}: \operatorname{Gal}\left(K / E_{j}\right) \rightarrow \mathbb{C}^{\times}$is the trivial representation. In particular we have $[K: M] \operatorname{dim} \phi=\sum_{j=1}^{v} m_{j}\left[E_{j}: M\right]$. Then

$$
\begin{aligned}
L\left(s,\left.\iota \rho_{\Pi}\right|_{\Gamma_{M}} \otimes \phi\right)^{[K: M]} & =\prod_{j=1}^{v} L\left(s,\left.\iota \rho_{\Pi}\right|_{\Gamma_{M}} \otimes \operatorname{Ind}_{\Gamma_{E_{j}}}^{\Gamma_{M}} 1_{E_{j}}\right)^{m_{j}} \\
& =\prod_{j=1}^{v} L\left(s, \operatorname{Ind}_{\Gamma_{E_{j}}}^{\Gamma_{M}}\left(\left.\iota \rho_{\Pi}\right|_{\Gamma_{E_{j}}}\right)\right)^{m_{j}} \\
& =\prod_{j=1}^{v} L\left(s,\left.\iota \rho_{\Pi}\right|_{\Gamma_{E_{j}}}\right)^{m_{j}} .
\end{aligned}
$$

Let $M_{j}:=E_{j} \cap M^{\prime}$. Then $E_{j}$ is a $(\mathbb{Z} / 2 \mathbb{Z})^{r_{j}}$-extension of $M_{j}$ for some $r_{j}$ (this is true because from the fact that $K$ is a $(\mathbb{Z} / 2 \mathbb{Z})^{r}$-extension of $M^{\prime}$, we get that $E_{j} M^{\prime}$ is a $(\mathbb{Z} / 2 \mathbb{Z})^{r_{j}}$-extension of $M^{\prime}$ for some $r_{j}$, and hence $E_{j}$ is a $(\mathbb{Z} / 2 \mathbb{Z})^{r_{j}}$-extension of $\left.M_{j}=E_{j} \cap M^{\prime}\right)$. Thus

$$
\begin{aligned}
L\left(s,\left.\iota \rho_{\Pi}\right|_{\Gamma_{M}} \otimes \phi\right)^{[K: M]} & =\prod_{j=1}^{v} L\left(s,\left.\iota \rho_{\Pi}\right|_{\Gamma_{E_{j}}}\right)^{m_{j}} \\
& =\prod_{j=1}^{v} \prod_{\phi_{j}: \operatorname{Gal}\left(E_{j} / M_{j}\right) \rightarrow \mathbb{C}^{\times}} L\left(s,\left.\iota \rho_{\Pi}\right|_{\Gamma_{M_{j}}} \otimes \phi_{j}\right)^{m_{j}} .
\end{aligned}
$$

Also one has

$$
\begin{aligned}
L\left(s,\left.\iota \rho_{\Pi}\right|_{\Gamma_{M}} \otimes \psi\right)^{[K: M]} & =L\left(s,\left.\iota \rho_{\Pi}\right|_{\Gamma_{M}} \otimes \phi \otimes \chi\right) \\
& =\prod_{j=1}^{v} \prod_{\phi_{j}: \operatorname{Gal}\left(E_{j} / M_{j}\right) \rightarrow \mathbb{C}^{\times}} L\left(s,\left.\left.\iota \rho_{\Pi}\right|_{\Gamma_{M_{j}}} \otimes \phi_{j} \otimes \chi\right|_{\Gamma_{M_{j}}}\right)^{m_{j}}
\end{aligned}
$$


Hence the function $L\left(s,\left.\iota \rho_{\Pi}\right|_{\Gamma_{M}} \otimes \psi\right)^{[K: M]}$ has a meromorphic continuation to the entire complex plane and satisfies a functional equation because from $\S 3$ we know that each function $L\left(s,\left.\left.\iota \rho_{\Pi}\right|_{\Gamma_{M_{j}}} \otimes \phi_{j} \otimes \chi\right|_{\Gamma_{M_{j}}}\right)$ has a meromorphic continuation to the entire complex plane and satisfies a functional equation. Also, since each function $L\left(s,\left.\left.\iota \rho_{\Pi}\right|_{\Gamma_{M_{j}}} \otimes \phi_{j} \otimes \chi\right|_{\Gamma_{M_{j}}}\right)$ has no poles or zeros for $\operatorname{Re}(s) \geqslant\left(k_{0}+1\right) / 2$, we get that the function $L\left(s,\left.\iota \rho_{\Pi}\right|_{\Gamma_{M}} \otimes \psi\right)$ has no poles or zeros for $\operatorname{Re}(s) \geqslant\left(k_{0}+1\right) / 2$. Thus for any integer $m$ satisfying

$$
k_{0}+1 \leqslant m
$$

we get the identity

$$
L\left(m,\left.\iota \rho_{\Pi}\right|_{\Gamma_{M}} \otimes \psi\right)^{[K: M]}=\prod_{j=1}^{v} \prod_{\phi_{j}: \operatorname{Gal}\left(E_{j} / M_{j}\right) \rightarrow \mathbb{C}^{\times}} L\left(m,\left.\left.\iota \rho_{\Pi}\right|_{\Gamma_{M_{j}}} \otimes \phi_{j} \otimes \chi\right|_{\Gamma_{M_{j}}}\right)^{m_{j}} .
$$

From $\S 3$ we know that

$$
L\left(m,\left.\left.\iota \rho_{\Pi}\right|_{\Gamma_{M_{j}}} \otimes \phi_{j} \otimes \chi\right|_{\Gamma_{M_{j}}}\right) \sim \pi^{\left(m+1-k_{0}\right)\left[M_{j}: \mathbb{Q}\right] \operatorname{dim} \chi}\langle f, f\rangle^{\frac{\left[M_{j}: F\right]}{2} \operatorname{dim} \chi},
$$

for any integer $m$ satisfying

$$
\left(k_{0}+1\right) / 2 \leqslant m<\left(k_{0}+k^{0}\right) / 2 .
$$

Hence from $[K: M] \operatorname{dim} \phi=\sum_{j=1}^{v} m_{j}\left[E_{j}: M\right]$ we get that

$$
\begin{aligned}
& L\left(m,\left.\iota \rho_{\Pi}\right|_{\Gamma_{M}} \otimes \psi\right)^{[K: M]}=\prod_{j=1}^{v} \prod_{\phi_{j}: \operatorname{Gal}\left(E_{j} / M_{j}\right) \rightarrow \mathbb{C} \times} L\left(m,\left.\left.\iota \rho_{\Pi}\right|_{\Gamma_{M_{j}}} \otimes \phi_{j} \otimes \chi\right|_{\Gamma_{M_{j}}}\right)^{m_{j}} \\
& \sim \pi^{\sum_{j=1}^{v}\left(m+1-k_{0}\right)\left[E_{j}: \mathbb{Q}\right] m_{j} \operatorname{dim} \chi}\langle f, f\rangle^{\sum_{j=1}^{v} \frac{\left[E_{j}: F\right]}{2} m_{j} \operatorname{dim} \chi} \\
& \sim \pi^{\left(m+1-k_{0}\right)[K: \mathbb{Q}] \operatorname{dim} \psi}\langle f, f\rangle^{\frac{[K: F]}{2} \operatorname{dim} \psi},
\end{aligned}
$$

and thus

$$
L\left(m,\left.\iota \rho_{\Pi}\right|_{\Gamma_{M}} \otimes \psi\right) \sim \pi^{\left(m+1-k_{0}\right)[M: \mathbb{Q}] \operatorname{dim} \psi}\langle f, f\rangle^{\frac{[M: F]}{2} \operatorname{dim} \psi},
$$

for any integer $m$ satisfying

$$
\left(k_{0}+1\right) / 2 \leqslant m<\left(k_{0}+k^{0}\right) / 2 .
$$

This concludes the proof of Theorem 1.1.

\section{References}

[BGGT] T. Barnet-Lamb, T. Gee, D. Geraghty, R. Taylor, Potential automorphy and change of weight, preprint.

[BGHT] T. Barnet-Lamb, D. Geraghty, M. Harris, R. Taylor, A family of CalabiYau varieties and potential automorphy II, P.R.I.M.S. 47 (2011), 29-98. 
[CR] C.W. Curtis, I. Reiner, Methods of Representation Theory, Vol. I, Wiley, New York, 1981.

[S1] G. Shimura, Algebraic relations between critical values of zeta functions and inner products, Amer. J. Math. 104 (1983), 253-285.

[S2] G. Shimura, The special values of the zeta functions associated with Hilbert modular forms, Duke Math. J. 45 (1978), 637-679.

[T] R. Taylor, On Galois representations associated to Hilbert modular forms, Invent. Math. 98 (1989), 265-280.

[V1] C. Virdol, Non-solvable base change for Hilbert modular forms and zeta functions of twisted quaternionic Shimura varieties, Annales de la Faculte des Sciences de Toulouse 19 (2010), no. 3-4, 831-848.

[V2] C. Virdol, On the Birch and Swinnerton-Dyer conjecture for abelian varieties attached to Hilbert modular forms, Journal of Number Theory 131 (2011), no. 4, 681-684.

[V3] C. Virdol, On the critical values of L-functions of tensor product of base change for Hilbert modular forms, Journal of Mathematics of Kyoto University 49 (2009), no. 2, 347-357.

[V4] C. Virdol, Tate classes and poles of L-functions of twisted quaternionic Shimura surfaces, Journal of Number Theory 123 (2007), no. 2, 315-328.

Address: Cristian Virdol: Department of Mathematics, Yonsei University, South Korea.

E-mail: virdol@yonsei.ac.kr

Received: 17 August 2011; revised: 2 October 2012 
